\title{
Forme initiale de la courbe de survie des cellules irradiées $\left(^{*}\right)$
}

\author{
J. DUTREIX (**)
}

(Manuscrit reçu le 17 septembre 1975)

\begin{abstract}
RÉSUMÉ
La partie initiale de la courbe de survie correspond aux doses qui intéressent la Radiothérapie et la Protection. Sa forme a un grand intérêt pour l'analyse des mécanismes radiobiologiques de la mort cellulaire. Ce travail discute les différentes formes de courbe de survie qui apparaissent dans les données de la littérature en particulier la pente initiale, les expressions mathématiques de ces courbes, les mécanismes radiobiologiques qu'elles suggèrent.
\end{abstract}

\begin{abstract}
The initial part of cell survival curves is related to doses of interest to Radiotherapy and Radiation Health Protection. Their shape provides a valuable approach to the fundamental mechanisms of cell killing by radiation. In this paper the different shapes of cell survival curves after the published data and the initial slope particularly, the mathematical expressions related to these curves, the radiobiological mechanisms suggested by the shapes of the curves, are discussed.
\end{abstract}

\section{INTRODUCTION}

La radiobiologie quantitative des cellules de mammifères a débuté avec les travaux de PUCK et MARCus [26]. Ces auteurs établirent la première courbe de survie, c'est-à-dire la relation entre le taux de survie et la dose délivrée en une séance unique de brève durée, pour des cellules HeLa ( fig. 1) cultivées et irradiées in vitro.

(*) Conférence présentée au Séminaire de Radiobiologie, organisé par la Société française de radioprotection à Fontenay-aux-Roses, 29-30 avril 1975.

$\left(^{* *}\right)$ Institut Gustave-Roussy, 16 bis, avenue Paul-Vaillant-Couturier, 94800 Villejuif.

RADIOPROTECTION, VOL. $11-\mathrm{N}^{\circ} 1$ 
Depuis cette étude, de nombreuses courbes de survie ont été obtenues, sur des types cellulaires variés, par diverses méthodes. Le domaine dans lequel est établie la relation survie-dose dépend du type cellulaire et de la méthode expérimentale; il dépasse rarement des doses de quelques milliers de rads et des taux de survie inférieurs à $10^{-4}$; il est souvent beaucoup plus restreint.

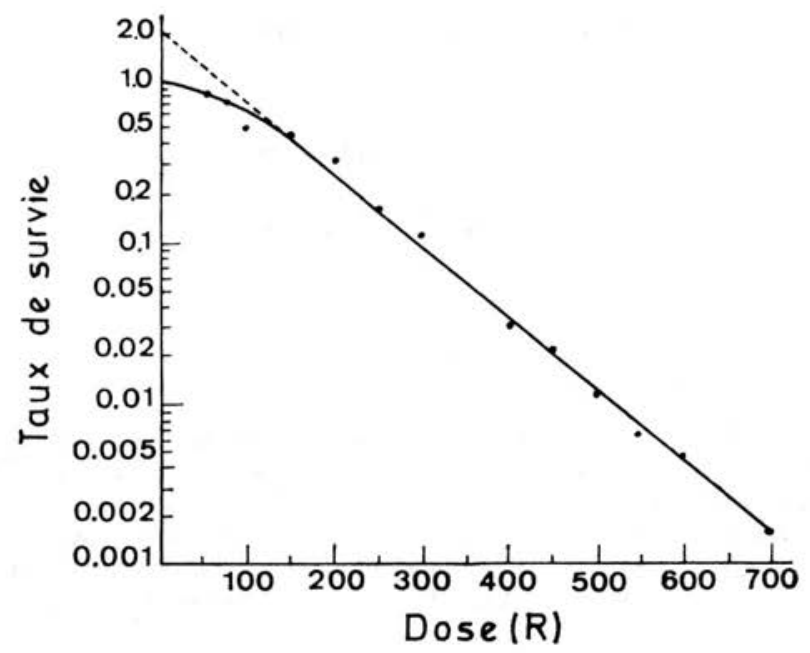

Fig. 1. - Courbe de survie des cellules HeLa, d'après Puck et Marcus, [26]

La forme la plus commune de la " courbe de survie " dans sa présentation habituelle (logarithme du taux de survie en fonction de la dose) est la courbe "à épaulement » dont la pente négative croît, en valeur absolue, avec la dose.

Les résultats expérimentaux suggèrent des différences dans la forme de ces courbes et leurs expressions mathématiques établies sur des modèles radiobiologiques diffèrent également.

La partie initiale de la courbe correspondant à des doses inférieures à quelques centaines de rads présente une importance particulière en radiothérapie, car les doses par séance les plus usuelles sont inférieures à 300 rads, et en radioprotection où les doses à considérer sont encore beaucoup plus faibles.

Par ailleurs, comme l'a souligné BARENDSEN [2] la partie initiale de la courbe peut refléter le rôle des différents mécanismes de la mort cellulaire.

Pour ces raisons, un intérêt particulier est porté depuis quelques années à la partie initiale de la courbe de survie [1].

La partie terminale de la courbe en reste cependant indissociable car elle concourt aux hypothèses radiobiologiques qui visent à interpréter l'ensemble de la courbe et à établir l'expression mathématique de celle-ci. 
Nous rappelons brièvement les méthodes expérimentales qui permettent d'étudier la relation survie-dose dans le domaine des doses relativement faibles. Nous discutons ensuite les différentes formes de courbes de survie qui se dégagent des résultats actuels. Nous verrons, enfin, les expressions mathématiques compatibles avec ces résultats et les modèles radiobiologiques sur lesquelles elles sont établies.

\section{MÉTHODES EXPÉRIMENTALES ET LEURS LIMITES}

\section{Méthodes DiRECTEMENT QUANTITATIVES}

Les méthodes de clonage in vitro [26] sont les plus courantes. Elles ont été appliquées à de nombreuses lignées de cellules malignes et normales, cultivables in vitro, l'irradiation étant faite in vivo ou in vitro.

TABLEAU

\begin{tabular}{c|c|c|c} 
& & \\
N Séances & \multicolumn{2}{c}{ Doses équivalentes } & \multicolumn{2}{c}{ Relation survie/dose } \\
& $\mathrm{D}$ rads & $\mathrm{S}(\mathrm{D})$ & $\log \mathrm{S}(\mathrm{D})$ \\
\hline 1 & 1400 & $\mathrm{~S}_{\mathrm{e}}$ & $\log \mathrm{S}_{\mathrm{e}}$ \\
2 & 850 & $\mathrm{~S}_{\mathrm{e}}^{1 / 2}$ & $1 / 2 \log \mathrm{S}_{\mathrm{e}}$ \\
4 & 515 & $\mathrm{~S}_{\mathrm{e}}^{1 / 4}$ & $1 / 4 \log \mathrm{S}_{\mathrm{e}}$ \\
8 & 290 & $\mathrm{~S}_{\mathrm{e}}^{1 / 8}$ & $1 / 8 \log \mathrm{S}_{\mathrm{e}}$
\end{tabular}

Les irradiations $N \times D$ sont équivalentes pour obtenir le mệme taux de survie final $S_{e}$ (le rôle de la multiplication cellulaire étant éliminé). Le taux de survie $S(D)$ correspondant à une dose $D$ est $S_{e} 1 / N$. Le logarithme de $S$, représenté sur la " courbe de survie " $\log S(D)$ est, pour la dose $D$, égal à $1 / N \log S_{e}$.

Elles permettent d'atteindre des taux de survie très faibles de $10^{-3}$ à $10^{-4}$ et, inversement, de garder une précision acceptable pour des taux de survie élevés supérieurs à 0,5 .

La méthode de clonage in vivo développée par WiTHERs [34] est une remarquable méthode pour l'étude des taux de survie de la peau et de la muqueuse intestinale (1969) mais elle ne fournit que la partie terminale de la courbe de survie pour des doses supérieures à 1000 rads.

La méthode de clonage splénique de McCulloch et Till [25] permet d'étudier le taux de survie des cellules médullaires clonogéniques dans un domaine de l'ordre de 0,5 à $10^{-2}$.

La méthode d'induction tumorale de HewitT et WILSON [20] est, en principe, utilisable pour toutes les tumeurs transplantables, mais elle présente 
des difficultés avec les tumeurs solides. Pour les leucémies, elle permet d'étudier la partie terminale de la courbe de survie jusqu'à des taux de survie très faibles de l'ordre de $10^{-5}$, mais elle paraît peu adaptée aux taux de survie supérieurs à $10^{-1}$.
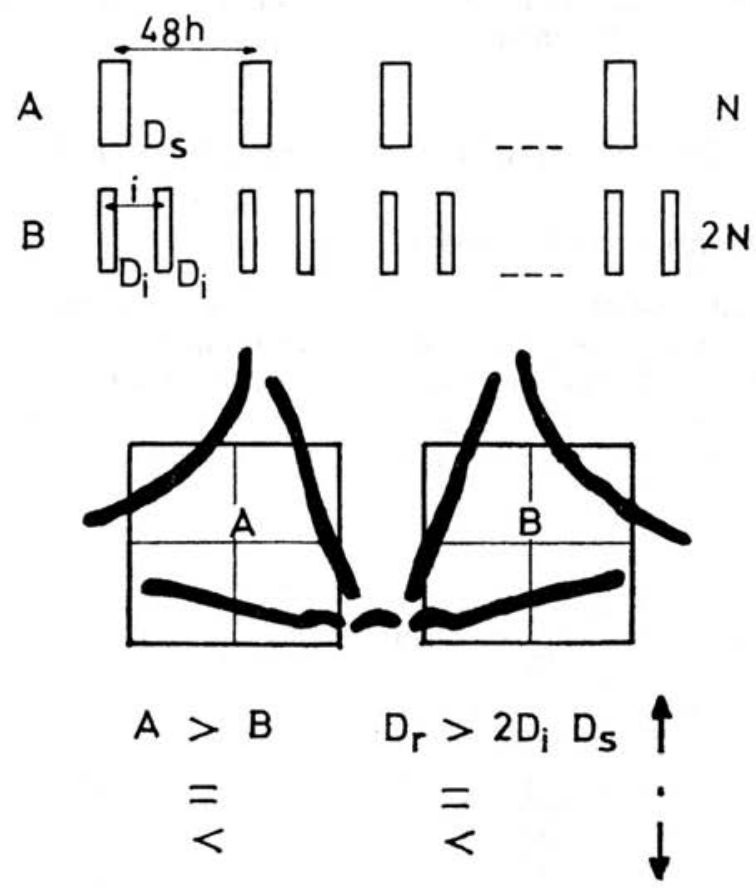

FIG. 2. - Les données cliniques sur lesquelles repose la construction de la courbe de survie cellulaire pour la peau proviennent de la comparaison des doses nécessaires pour obtenir la même réaction de desquamation sur deux champs symétriques irradiés en $N$ et $2 N$ séances étalées sur le même temps total (voir texte).

En résumé, la plupart des courbes de survie publiées ont été établies par clonage in vitro après irradiation in vivo ou in vitro. Leur précision sera discutée plus loin.

\section{MÉTHODE INDIRECTEMENT QUANTITATIVE}

Nous avons utilisé une méthode indirecte dans le but de déterminer la forme de la courbe de survie lorsque les cellules sont inaccessibles à une numération, ce qui est le cas habituel pour les études purement in vivo, et en particulier les études cliniques. La méthode a été initialement développée pour étudier les réactions de desquamation de la peau humaine [9]. Cet effet biologique n'est pas accessible à la mesure mais on peut définir l'égalité de l'effet sur deux champs cutanés symétriques irradiés chez le même sujet. 
On a comparé les doses nécessaires pour obtenir le même effet dans deux irradiations fractionnées en $N$ séances à dose $D_{s}$ et $2 N$ séances à dose $D_{i}$ et étalées sur la même durée totale ( fig. 2).

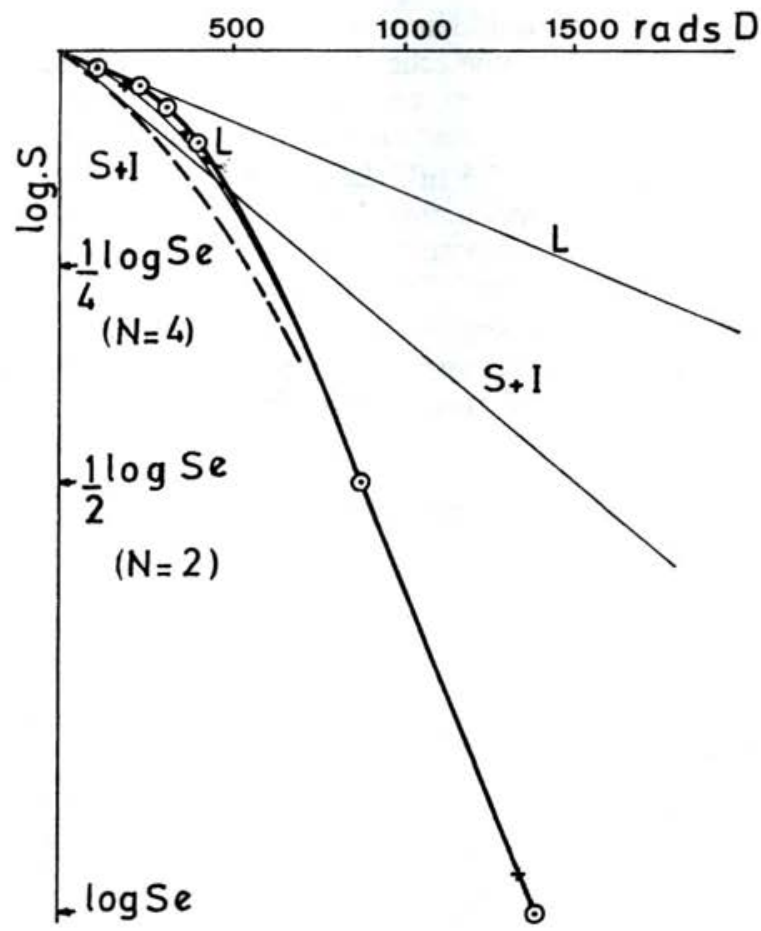

FIG. 3. - Forme des courbes de survie obtenues (avec la méthode schématisée à la partie supérieure de la figure 2) pour la peau humaine et la muqueuse intestinale (courbes $\mathrm{S}+\mathrm{I}$ en tiret) et le poumon (courbe $\mathrm{L}$ en trait plein). Les droites en trait fin représentent les tangentes initiales. L'échelle des ordonnées est arbitraire et les courbes sont normalisées à l'abscisse 1400 rads.

On accepte les trois hypothèses suivantes :

a) une égalité d'effet correspond à une égalité des taux de survie cellulaire;

b) la courbe de survie cellulaire se retrouve identique à elle-même à chaque séance d'irradiation [13];

c) la multiplication cellulaire est la même au cours des deux irradiations.

Si l'effet final est le même sur les deux champs, on conclut :

a) que l'effet d'une dose $D_{s}$ est identique à celui de deux doses $D_{i}$;

b) que l'accroissement de la dose lorsqu'une dose $D_{s}$ est remplacée par deux doses $D_{i}$ est uniquement dû à la restauration cellulaire, le rôle de la multiplication cellulaire étant éliminé $[10,11]$. 
Une série d'études cliniques a permis d'établir la correspondance entre $D_{s}$ et $2 D_{i}$ pour un domaine de dose de 300 à 1400 rads, en adaptant le nombre de séances aux doses par séance utilisées. On en déduit la relation entre le taux de survie et la dose schématisée sur le tableau.

Le taux de survie reste indéterminé mais la forme de la courbe de survie ( fig. 3) est définie avec une échelle d'ordonnées arbitraires [31]. Le taux de survie peut être déterminé si on adopte pour la pente de la courbe entre 850 et 1400 rads la valeur obtenue par WITHERs [34] soit $D_{0}=135$ rads.

La méthode précédente a été utilisée d'une part pour la muqueuse intestinale de la souris, l'effet final considéré étant la létalité après irradiation abdominale [32] et d'autre part pour le poumon de la souris, l'effet final étant la létalité à cent jours après irradiation pulmonaire [11].

Les courbes de survie correspondantes sont représentées en ordonnées arbitraires sur la figure 3 avec normalisation des courbes à 1400 rads. Elles montrent des différences de forme significatives.

\section{TYPES DE COURBES}

Dans la quantité de courbes de survie publiées, on peut schématiquement considérer quatre types que nous illustrerons par quelques exemples.

\section{TYPE EXPONENTIEL}

La courbe de survie exponentielle s'observe pour les systèmes biologiques les plus élémentaires comme les virus. La $D_{\mathrm{o}}$ (dose réduisant le taux de survie à $1 / e$ soit environ 0,37 ) est de 1 'ordre de quelques dizaines à quelques centaines de milliers de rads. Elle est en relation avec la masse moléculaire et en accord avec la relation générale observée pour l'inactivation de molécules biochimiques [24].

Le type exponentiel s'observe de façon inconstante pour des systèmes plus complexes : bactéries, levures haploïdes avec une $D_{0}$ de l'ordre de 1000 , 2000 rads. Il se rencontre plus rarement avec les cellules de mammifères, en particulier en phase de mitose [29].

C'est le type de courbe vers lequel tendent les courbes de survie de tous les systèmes biologiques pour des rayonnements de TEL croissant [3].

Par ailleurs, certains agents chimiques (caféine) ou physique (chaleur) tendent à donner à la courbe de survie une forme exponentielle; nous reviendrons (§ IV-2) sur le rôle de ces agents.

\section{Courbes avec ÉPAulement}

Pour les cellules de mammifères, la plupart des résultats expérimentaux sont incompatibles avec une courbe exponentielle et impliquent une incurvation de la courbe de survie.

Le tracé de la courbe à travers les points expérimentaux affectés de leur barre d'erreur peut se faire en présupposant que la relation survie-dose 
correspond libres. Les méthodes statistiques de " lissage " permettent de définir les valeurs numériques les plus probables des paramètres de la fonction [27] mais elles ne démontrent pas la validité du choix de la fonction.

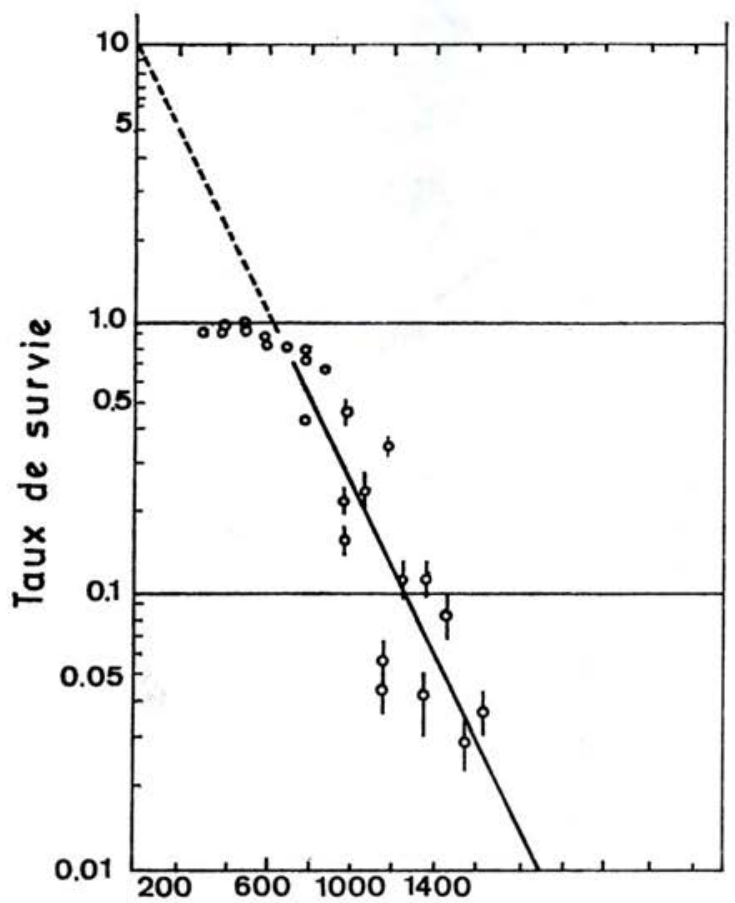

Fig. 4. - Courbe de survie des mélanoblastes dan; le follicule pileux, d'après C. S. Potten et H. B. ChASE, Rad. Res., 1970, 42, 305-319.

Le tracé à l'œil est apparemment moins contraignant, cependant il est guidé par un choix plus ou moins conscient dans la forme de la courbe : la figure 4 donne un exemple d'un tracé qui est dicté par l'hypothèse que la fonction est exponentielle entre les taux de survie 0,5 et la dernière valeur expérimentale 0,03 .

La courbe de survie avec épaulement est généralement exprimée par les deux paramètres $D_{0}$ et $n$ correspondant à la pente finale et au nombre d'extrapolation, ce qui suppose que la partie terminale de la courbe est exponentielle. Cette hypothèse est plausible pour certaines études qui fournissent un nombre de points expérimentaux alignés sur une exponentielle dans un large domaine de dose et de taux de survie ( $f$ ig. 1). La signification des paramètres $D_{0}$ et $n$ est, par contre, très douteuse lorsque la construction de l'exponentielle terminale repose sur une partie peu étendue de la courbe ( $f i g .5$ ). Selon toute probabilité, cette construction surestime $D_{0}$ et sous-estime $n$. 
De façon plus générale l'hypothèse d'une asymptote exponentielle est alors arbitraire. Certaines courbes ( fig. 6) présentent une incurvation continue dans tout le domaine des valeurs expérimentales.

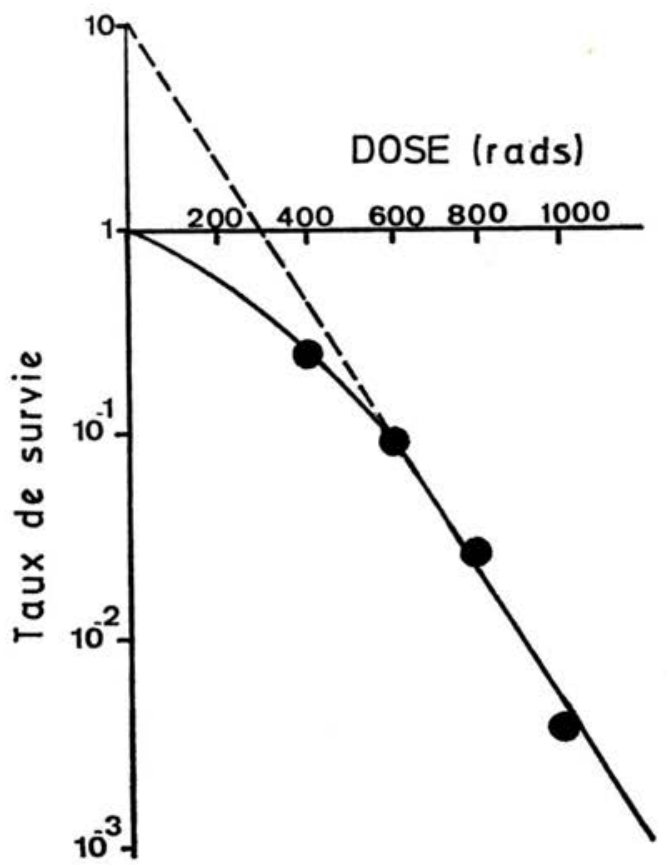

FIG. 5. - Courbe de survie des cellules de la poche jugale du Hamster, d'après; BeRRY [5].

Les résultats expérimentaux publiés dans la littérature présentent des différences importantes de la forme initiale de la courbe de survie. Certains montrent que les faibles doses provoquent une mortalité cellulaire inappréciable et suggèrent que la tangente initiale à la courbe de survie est horizontale (fig. 4).

D'autres, au contraire, montrent un effet appréciable avec des doses de quelques dizaines de rads et suggèrent que la tangente à l'origine présente une certaine pente sans toutefois pouvoir l'affirmer, c'est-à-dire prévoir l'effet d'une très petite dose de l'ordre de quelques rads.

En théorie, l'utilisation d'un très grand nombre de très petites doses espacées ou d'une irradiation continue à très faible débit devrait permettre d'approcher la tangente initiale de la courbe de survie. L'irradiation continue fait apparaître d'autres phénomènes attribués à un blocage des cellules en phase radiosensible qui conduisent à des résultats paradoxaux [12].

On ne peut donc pas considérer que la courbe de survie obtenue avec une irradiation continue à très faible débit représente la tangente initiale 
de la courbe de survie pour une irradiation aiguë. Cependant, le fait que la dose totale nécessaire pour obtenir un effet déterminé avec une irradiation continue ne croît pas indéfiniment lorsque le débit diminue [16] est un solide argument pour admettre que la courbe de survie de la population étudiée présente une pente initiale.

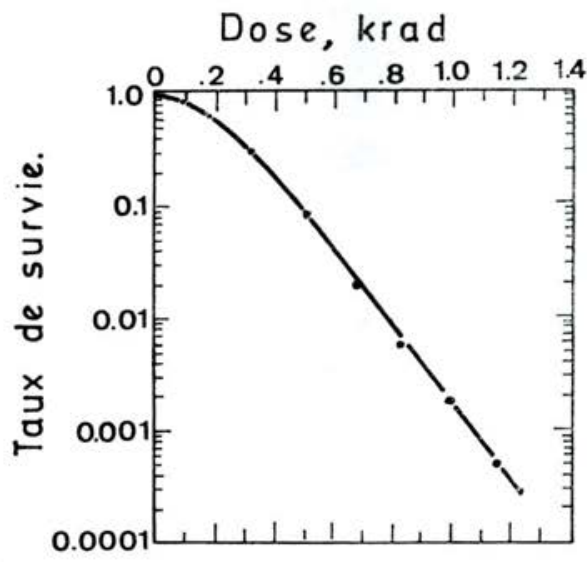

B

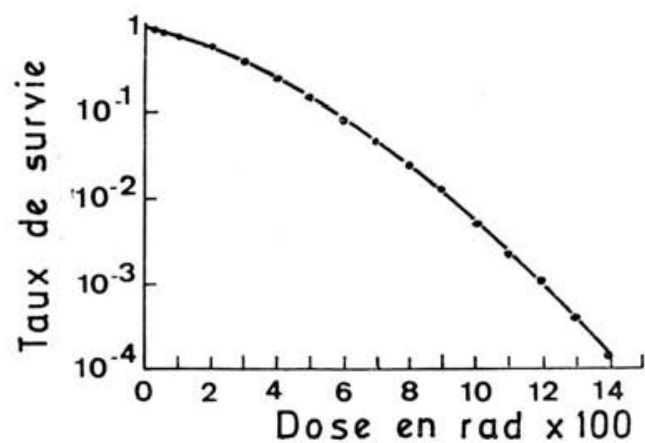

C

Fig. 6. - Comparaison de deux courbes de survie établies dans un domaine comparable de doses et de taux de survie et qui présentent une partie terminale exponentielle (B) ou une incurvation continue (C) (d'après E. J. HALL, in Radiobiology for the radiologist, Hagerstown, Harper and Row, 1973, 21).

Courbe B : cellules de Hamster (Elkind and Sutton) [13].

Courbe $\mathrm{C}$ : cellules de rein humain (Barendsen) [2].

\section{Courbe DE SURVIE AVEC SEUIL}

Bien que ce type de courbe soit plus rare que les précédents, il faut mentionner que pour certaines courbes de survie, l'irradiation paraît totalement dénuée d'effet tant qu'elle n'atteint pas une valeur seuil pour laquelle la mortalité cellulaire commence à se manifester.

VOL. $11-\mathrm{N}^{\circ} 1$ 
La figure 4 évoque ce type de courbe pour des cellules de mammifères. D'autres, plus démonstratives, ont été publiées pour des systèmes biologiques végétaux $[16,17]$. Ces courbes semblent bien représenter un type distinct des courbes à épaulement avec tangente initiale de pente nulle.

En définitive, on peut admettre l'existence des quatre types de courbes schématisées sur la figure 7 :

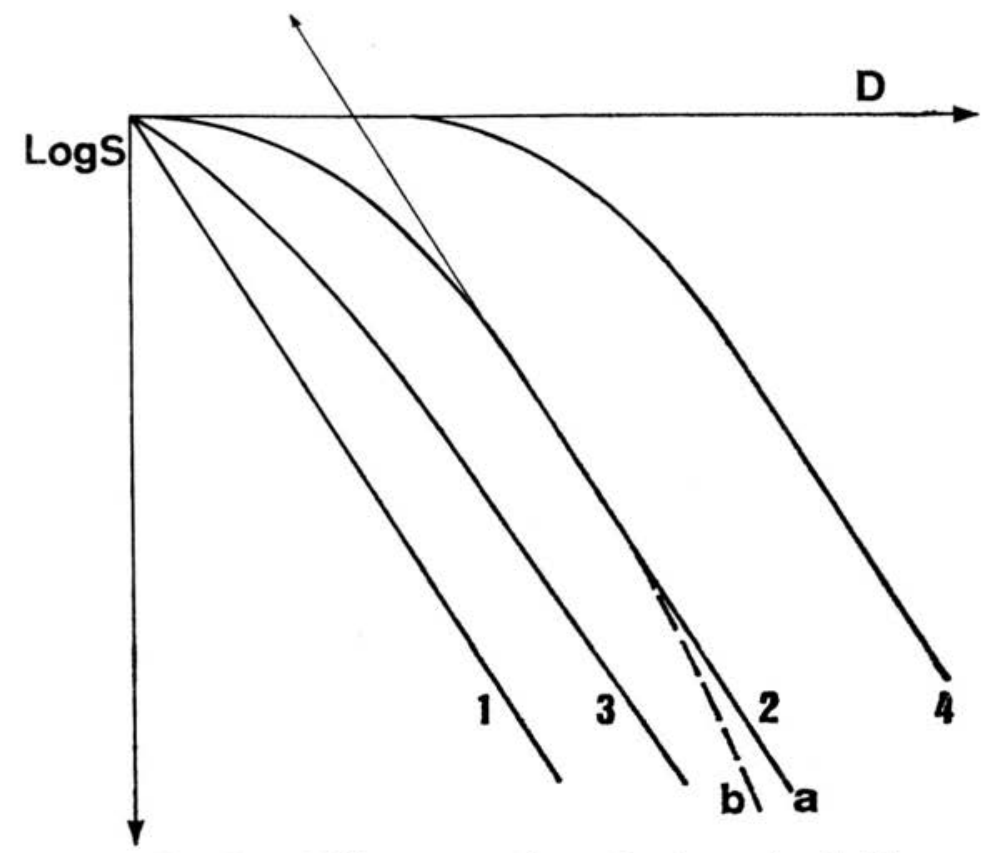

Fig. 7. - Différents types de courbes de survie cellulaire.

$1^{\circ}$ exponentielle;

$2^{\circ}$ avec épaulement et tangente initiale de pente nulle;

$3^{\circ}$ avec épaulement et tangente initiale de pente non nulle;

$4^{\circ}$ avec épaulement et avec une dose seuil.

Pour les types 2,3 et 4 , deux variantes concernent la partie terminale (figurées seulement sur la courbe 2).

a) asymptote exponentielle;

b) pente continuellement croissante en valeur absolue.

Ces quatre types de courbes ont été observées par HollaENDER [21] pour une même espèce cellulaire $(E$. Coli) :

$1^{\circ}$ bactéries en bouillon de culture aérobie;

$2^{\circ}$ bactéries en bouillon de culture anaérobie;

$3^{\circ}$ bactéries cultivées sur glucose aérobie;

$4^{\circ}$ bactéries cultivées sur glucose anaérobie. 


\section{INTERPRÉTATION BIOLOGIQUE ET EXPRESSIONS MATHÉMATIQUES}

Il est admis que la mort cellulaire, c'est-à-dire la perte de l'aptitude à la réplication cellulaire indéfinie, est essentiellement liée à l'atteinte de l'ADN par les rayonnements et plus spécialement aux ruptures du squelette phosphoglucidique qui peuvent intéresser l'un des deux filaments de la double hélice ou les deux.

Les doubles ruptures produites simultanément par le passage d'une particule au même niveau de la molécule d'ADN provoquent des altérations de la fonction de l'ADN qui entraînent la mort de la cellule. Ces lésions sont irréversibles pour FreIFELDER et SzYBALSKY $[15,29]$ tandis que HowARD-FLANDERS [23] est conduit à conclure que la plupart sont réparables; dans ce dernier cas, l'événement létal est la double rupture non réparée.

Les ruptures simples ont individuellement des conséquences moins graves, car elles sont réparables et la molécule d'ADN retrouve son intégrité. Cependant, l'accumulation de ruptures simples pendant l'irradiation peut aboutir à la dénaturation de la molécule et à la mort de la cellule. En particulier, une deuxième rupture simple en regard d'une rupture qui n'est pas encore réparée est équivalente à une rupture double d'emblée.

On peut donc considérer que la mort cellulaire peut résulter d'un événement qui, d'emblée, condamne la cellule ou de l'accumulation d'événements " sublétaux » individuellement non mortels. La mort de la cellule dépend donc de la probabilité de production des liaisons élémentaires et, par ailleurs, de la probabilité de leur réparation avant leur expression létale pour la cellule.

La plupart des modèles cellulaires utilisés pour exprimer la relation survie-dose pour une irradiation unique considèrent essentiellement la probabilité de production de ces événements. Nous discuterons un modèle dans lequel le rôle principal est attribué à leur réparation.

\section{Modèles BalistiQues}

Les événements létaux d'emblée ont pour traduction une courbe de survie exponentielle. Si $a$ représente la probabilité, rapportée à 1 rad, pour qu'un événement létal se produise dans une cellule, le taux de survie est représenté par $\mathrm{S}=e^{-a}{ }^{\mathrm{l}} \mathrm{ou}$, en posant $D_{1}=1 / a$ :

$$
S=e^{-D / D_{1}}
$$

$D_{1}$ représente la dose pour laquelle il y a, en moyenne, un événement létal par cellule (c'est-à-dire $N$ événements létaux dans une population de $N$ cellules). Dans ces conditions une fraction $1 / e=37 \mathrm{p}$. cent des cellules ne présente aucun événement létal et survit tandis que le complément ( $63 \mathrm{p}$. cent) représente la fraction des cellules dans lesquelles se produisent un ou plusieurs événements létaux et qui sont donc tuées.

vOL. $11-\mathrm{N}^{\circ} 1$ 
La mort par accumulation d'événements sublétaux peut répondre à deux mécanismes différents qui conduisent à des expressions différentes de la survie en fonction de la dose.

- On peut considérer que la mort cellulaire résulte en moyenne de la production de $n$ événements sublétaux aléatoires (par exemple $n$ ruptures simples quelconques).

Si on désigne par $k$ la probabilité (supposée uniforme) de la production d'un événement sublétal la relation survie-dose prend la forme

$$
S=1-\left(1-e^{-k D}\right)^{n},
$$

ou en posant $D_{n}=1 / k$ :

$$
S=1-\left(1-e^{-D / D_{n}}\right)^{n}
$$

$D_{n}$ représente la dose pour laquelle il y a en moyenne un événement sublétal par cellule.

La courbe de survie (log $S$ en fonction de $D$ ) présente une tangente initiale de pente nulle et a pour asymptote l'exponentielle $n e^{-D / D_{n}}$.

- On peut considérer que la mort cellulaire résulte de l'accumulation de deux événements sublétaux à l'intérieur d'un volume élémentaire de dimensions finies (c'est-à-dire, par exemple, de la production de deux ruptures simples indépendantes sur les deux filaments de la molécule d'ADN et à une distance suffisamment faible pour réaliser une rupture double).

La relation survie-dose est alors exprimée par la relation

$$
S=e^{-b D^{2}} .
$$

La signification balistique du paramètre $b$ a été développée par CHADWICK et LEEHNOUTS [7].

Les courbes de survie correspondant aux relations (2) et (3) ont une pente initiale nulle. Elles supposent que la mortalité cellulaire résulte uniquement de l'accumulation d'événements sublétaux. Si l'on admet la coexistence de mort cellulaire par événements létaux d'emblée, c'est-à-dire un double mécanisme de mort cellulaire, la relation survie-dose est le produit de l'expression (1) : taux de survie relatif aux événements létaux d'emblée - et de l'expression (2) ou (3) : taux de survie relatif à l'accumulation d'événements sublétaux soit

$$
\begin{aligned}
S & =e^{-D / D_{1}}\left[1-\left(1-e^{-D / D_{n}}\right)^{n}\right], \\
\text { ou } \quad S & =e^{-\left(a D+b D^{2}\right)} \quad \text { avec } \quad a=\frac{1}{D_{1}} .
\end{aligned}
$$

En résumé :

a) l'existence d'événements létaux d'emblée implique que la courbe de survie présente une pente à l'origine; 
b) la possibilité de mort cellulaire par accumulation d'événements sublétaux explique l'incurvation de la courbe de survie.

Dans les deux modèles précédents, la mortalité par ce deuxième mécanisme tend vers zéro pour les doses de plus en plus faibles et seul le premier mécanisme serait alors en cause.

Les courbes " avec seuil " s'interprètent mal avec le modèle balistique. Elles impliquent évidemment l'absence d'événements létaux d'emblée, mais par ailleurs l'apparition rapide de la mortalité cellulaire au-delà de la dose seuil est peu compatible avec la statistique des événements sublétaux.

\section{Modèle enZyMatiQue}

Le rôle des mécanismes de réparation cellulaire dans la relation taux de survie-dose a été mis en évidence sur de nombreux systèmes biologiques.

L'absence ou la déficience du système de réparation augmente la radiosensibilité. Elle peut être génétique : les mutants de $E$. Coli déficients en DNA-polymérase ont une radiosensibilité élevée [30]; dans le Xeroderma pigmentosum, CLEAVER [8] a mis en évidence la déficience du système de réplication de l'ADN.

La radiosensibilité est augmentée par des agents physiques comme la chaleur [4], ou chimiques comme la caféine [6], qui inhibent les enzymes de réparation et par des substances comme l'actinomycine $\mathrm{D}$ et la 5.bromodeoxyuridine, qui interfèrent dans le processus biochimique de réplication.

La forme des courbes de survie peut être rapportée au mécanisme de restauration selon le schéma suivant : L'irradiation entraîne des événements potentiellement létaux qui fixent des unités du pool enzymatique de réparation. La fraction du pool disponible par événement diminue à mesure que la dose augmente et il en résulte une diminution de la probabilité de la réparation des événements.

Cet épuisement du système de réparation peut être mis en évidence expérimentalement [33].

L'épaulement de la courbe de survie peut s'expliquer ainsi par l'épuisement du pool; le taux de mortalité cellulaire représenté par la pente de la tangente à la courbe croît lorsque la dose augmente. Lorsque le pool est totalement épuisé, tous les événements restent non réparés et deviennent létaux. La courbe de survie devient exponentielle dans sa partie terminale (la pente représente alors le taux de production des événements qui est égal au taux de mortalité cellulaire). Si l'épuisement reste incomplet pour la plus grande dose utilisée, la courbe présente une incurvation continue dans le domaine étudié et la terminaison exponentielle n'apparaît pas.

Lorsque la courbe a une tangente initiale de pente nulle, on peut admettre que les événements sont tous potentiellement réparables et que les premiers événements sont effectivement tous réparés; la diminution du pool résultant de la fixation des unités enzymatiques entraîne aussitôt une diminution de la probabilité de réparation lorsque la dose augmente.

voL. $11-\mathrm{N}^{\circ} 1$ 
Lorsque la courbe a une tangente initiale de pente non nulle on peut admettre qu'une fraction des événements est d'emblée irréparable.

Une courbe avec seuil peut s'interpréter en considérant que les événements sont tous potentiellement réparables et qu'ils sont tous réparés tant que le pool reste supérieur à une valeur critique; celle-ci est atteinte pour une dose telle que l'accumulation des événements a abaissé le pool au niveau de la valeur seuil. Au-delà de cette dose, l'épuisement progressif du pool se manifeste comme dans les cas précédents et le taux de mortalité cellulaire s'élève à mesure que la dose croît.

La courbe de survie exponentielle correspond au cas où tous les événements restent non réparés, soit qu'ils se trouvent d'emblée irréparables, soit que, potentiellement réparables, ils n'aient pas à leur disposition de système de réparation actif.

Ainsi, le modèle enzymatique permet d'interpréter toutes les formes connues des courbes de survie. Il se distingue essentiellement des « modèles balistiques " en substituant au concept d'atteintes sublétales dont l'accumulation devient létale, la notion d'atteintes potentiellement létales et potentiellement réparables qui deviennent létales lorsque le système de réparation n'assure pas totalement sa fonction.

Un modèle mathématique construit sur ce mécanisme biologique a été développé par CALKINS et HAYNES $[6,10]$.

\section{BIBLIOGRAPHIE}

[1] AlPER T. The initial shape of cell survival curves. L. H. Gray Conference, London, September 1974, Londres, J. Wiley, 1975.

[2] Barendsen G. W. Dose-survival curves of human cells in tissue culture irradiated with alpha, beta, 20-kV X and $200 \mathrm{kV}$ X-radiation. Nature, 1962, 193, 1153.

[3] Barendsen G. W. The effect of oxygen on impairment of the proliferative capacity of human cells in culture by ionizing radiations of different LET. Int. J. Rad. Biol., 1966, 10, 317.

[4] Ben HUR E., ElKInd M. M., BRONK B. V. Thermally enhanced radioresponse of cultured chinese Hamster cells: inhibition of repair of sublethal damage and enhancement of lethal damage. Rad. Res., 1974, 58, 38.

[5] Berry R. J. Population distribution in tumors and normal tissues. In The biological and clinical basis of radiosensitivity (M. Friedman Ed.), Springfield, C. C. Thomas, 1974.

[6 Calkins J. A method of analysis of radiation response based on enzyme kinetics. Rad. Res., 1971, 45, 50.

[7] Chadwick K. H., Leenhouts H. P. A molecular theory of cell survival. Phys. Med. Biol., 1973, 18, 78-87.

[8] Cleaver J. E. Defective repair replication of DNA in Xeroderma pigmentosum. Nature, 1968, 218, 652.

[9] Dutreix J., Wambersie A., BouniK C. Cellular recovery in human skin reactions: application to dose, fraction number, overall time relationship in radiotherapy. Europ. J. Cancer, 1973, 9, 159-167. 
[10] Dutreix J., Wambersie A. Cell survival curves deduced from non quantitative reactions of skin, intestinal mucosa and lung, L. H. Gray Conference, London, September 1974, Londres, J. Wiley, 1975.

[11] Dutreix J., Wambersie A. Iso effect total dose as a function of the number of fractions for skin, intestinal mucosa and lung. Proceedings of the conference on time-dose relationships in clinical therapy, Madison. University of Wisconsin, October 1974.

[12] Dutreix J., Sahatchiev A. Clinical radiobiology of low dose rate radiotherapy. Brit. J. Radiol., 1975, 48, 846-850.

[13] ElKind M. M., SutToN H. Radiation response of mammalian cells grown in culture. I. Repair of X-ray damage in surviving Chinese hamster cells. Rad. Res., 1960, 13, 556-593.

[14] Fowler J. F., STERn B. E. Dose-time relationships in radiotherapy and validity of cell survival curve models. Brit. J. Radiol., 1963, 36, 163.

[15] Freifelder D. Lethal changes in bacteriophage DNA produced by X-ray. Rad. Res., 1966, suppl. 6, 80.

[16] HaLl E. J. Radiation dose-rate: a factor of importance in radiobiology and radiotherapy. Brit. J. Radiol., 1972, 45, 81.

[17] Hall E. J. Radiobiology for the radiologist, Hagerstown, Harper and Row, 1973, p. 21.

[18] HAYNES R. H. The interpretation of microbial inactivation and recovery phenomena. Rad. Res., 1966, suppl. 6, 1.

[19] HaYNes R. N. The influence of repair processes in radiobiological survival curves. L. H. Gray Conference, London, September 1974, Londres, J. Wiley, 1975.

[20] Hewitr H. B., Wilson C. W. A survival curve for mammalian cells irradiated in vivo. Nature, 1959, 183, 1060-1061.

[21] Hollaender A., Stapleton G. E., Martin F. L., Nature, 1951, 167, 103.

[22] Howard A. Survival curve characteristics in a Desmid. L. H. Gray Conference, London, September 1974, Londres, J. Wiley, 1975.

[23] Howard-Flanders P. Variation of radiosensitivity and recovery from radiation effects in genetic structures. In The Biological and Clinical Basis of Radiosensitivity (Friedman M. Ed.), Springfield, C. C. Thomas, 1974, 101-114.

[24] Kaplan H. S., Moses L. E. Biological complexity and radiosensitivity. Science, 1964, 145, 21.

[25] MCCulloch E. A., Till J. E. The sensitivity of cells from normal mouse bone marrow to gamma radiation in vitro and in vivo. Rad. Res., 1962, 16, 822-832.

[26] Puck T. T., Marcus P. I. Action of X-ray on mammalian cells. J. Exp. Med., 1956, 103, 653-666.

[27] Roux J.-C. Irradiation de chlorelles par des rayonnements particulaires. Thèse, Grenoble, 1974.

[28] Sinclair W. K. Cyclic X-ray responses in mammalian cells in vitro. Rad. Res., 1968, 33, 620-643.

[29] SzYBALSKı W. Molecular events resulting in radiation injury, repair and sensitization of DNA. Rad. Res., 1967, suppl. 7, 147.

[30] Town C. D., Smith K. C., Kaplan H. S. DNA polymerase required for rapid repair of X-ray induced strand breaks in vivo. Science, 1971, 172, 851.

[31] Wambersie A., Dutreix J., Gueuletre J. Determination of the shape of mammalian cell survival curves by the evaluation of cellular recovery as a function of the size of the fraction. In Proceedings of the 4th Symposium on Microdosimetry, Verbania Pallanza, September 1973.

VoL. $11-\mathrm{N}^{\circ} 1$ 
[32] Wambersie A., Dutreix J., Gueulette J., Lellouch J. Early recovery for intestinal stem cells, as a function of dose per fraction, evaluated by survival rate after fractionated irradiation of the abdomen of mice. Rad. Res., 1974, 58, 498.

[33] Waters R., Moustacchi E. Dose dependence of the excision of ultraviolet induced pyrimidine dimers from nuclear deoxyribonucleic acids haploid and diploid Saccharomyces cerevisiae. J. Bacteriol., 1975, 121, 3.

[34] Wrthers H. R. The dose-survival relationship for irradiation of epithelial cells of mouse skin. Brit. J. Radiol., 1967, 40, 187.

[35] WITHERS H. R., ElKIND M. M. Radiosensitivity of fractionation in response of crypt cells of mouse jejunum. Rad. Res., 1969, 38, 598. 